\title{
Unusual complication after laparoscopic left nephrectomy for renal tumour: a case report
}

\author{
Arantxa Arruabarrena', Juan Santiago Azagra², Jean Francoise Wilmart², loan Bachner², Dan Manzoni' ${ }^{2}$ Martine Goergen² \\ ${ }^{1}$ Hospital Universitario Marqués de Valdecilla, Spain \\ ${ }^{2}$ Centre Hospitalier de Luxembourg, Luxembourg
}

Videosurgery and other miniinvasive techniques 2010; 5 (2): 60-64

DOI: 10.5114/wiitm.2010.14205

\begin{abstract}
In splenic rupture after blunt trauma, iatrogenic spleen injury or non-traumatic cases it is essential that the surgeon makes correct decisions. Conservative treatment must include continual monitoring and repeated, stringent evaluation of the splenic injury (the American Association for the Surgery of Trauma-AAST) in order to avoid any delay in diagnosis of delayed spleen rupture and the high mortality it causes. We present the case of an unexpected complication after radical nephrectomy performed for renal cell carcinoma. A 61-year old man sought medical help for acute abdominal pain. He presented with some cardiovascular risk factors (diabetes mellitus, smoker of 30 cigarettes per day) and moderate alcohol use. In the Emergency Unit, computed tomography scan revealed an incidental tumour of the left kidney. Nephrectomy via the laparoscopic approach was done without any iatrogenic complications, with less than $500 \mathrm{cc}$ of blood loss. Firm adhesions between the spleen and abdominal wall, which caused some minor traction that resulted in a small subcapsular haematoma, were the only surprising intraoperative finding. Within the first $6 \mathrm{~h}$, the patient presented with haemodynamic instability, while the drain evacuated less than $50 \mathrm{cc}$ of discharge. However, CT scan showed that subcapsular haematoma had increased to the size of $10 \times 10 \mathrm{~cm}$ without free peritoneal fluid present. Unfortunately, $6 \mathrm{~h}$ later emergency surgery had to be performed due to rupture of the subcapsular splenic haematoma. Massive haemoperitoneum was evacuated and the splenic capsule was the only remnant of the spleen that could be found on re-intervention. So far, it is the first case describing an increasing subcapsular haematoma of the spleen, most likely caused by the traction of firm adhesions to the organ. We discuss means to avoid such a complication: with liberation of the adhesions, placement of a perisplenic mesh, embolization of the splenic artery or subcapsular nephrectomy. An acute splenic rupture or delayed one demands from the surgeon practical knowledge of how to prevent subcapsular haematoma and how to treat splenic rupture.
\end{abstract}

Key words: delayed splenic rupture, subcapsular haematoma, nephrectomy.

\section{Introduction}

Delayed splenic rupture was first described by Baudet in 1902. Today it is defined as a late occurrence of signs and symptoms attributed to splenic injury, which was not detected by diagnostic computed tomographic (CT) scanning during an initial examination [1].
The significance of delayed splenic rupture is related to its high mortality rate $(5-15 \%)$ due to late diagnosis. High index of suspicion and liberal use of imaging techniques are essential for diagnosis of delayed splenic rupture. Thorough evaluation of the degree of splenic injury with the Splenic Injury Scale, 
as proposed by the American Association for the Surgery of Trauma (AAST), is a must. The presence of subcapsular haematoma, pseudocyst, or pseudoaneurysm must be taken into consideration [1].

Several hypotheses have tried to explain the mechanism of rupture. Baudet et al. suggested that blunt abdominal injury often carries enough force to damage the splenic parenchyma, without breaking the capsule. Once intrasplenic bleeding commences, subcapsular haematoma is a major risk factor for rupture and haemoperitoneum [2].

However, all papers found in the literature describe delayed splenic injury resultant from a blunt trauma, iatrogenic damage during adhesiolysis or occurring in pathological spleens, for instance in infectious mononucleosis. We have not found a single report that relates to a delayed spleen rupture without physical contact with the organ and due to local and gravitational factors only.

Our objective is to present an unexpected and rare case of morbidity after laparoscopic surgery.

\section{Case report}

A 61-year old man with cardiovascular risk factors including diabetes mellitus and smoking of 30 cigarettes a day and moderate alcohol use was admitted for acute abdominal pain in the epigastric area.

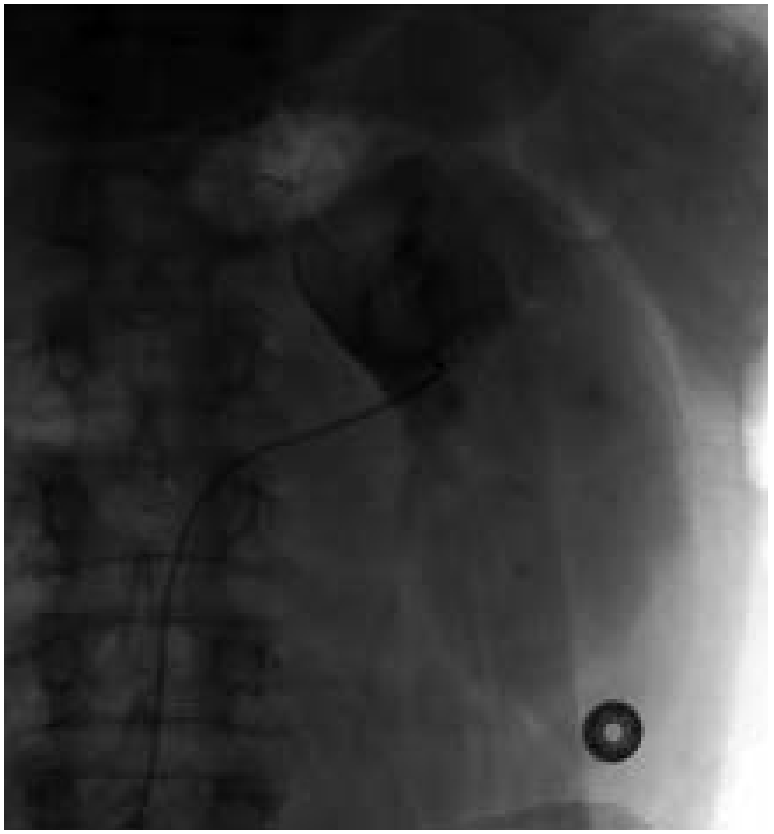

Figure 1. Renogram of the left kidney
Urgent electrocardiogram and coronarography were performed for suspicion of cardiac ischaemia. Nevertheless, the complementary tests and cardiac enzymes proved to be normal.

The patient also reported back pain and some blood in the stools. Colonoscopy was performed, and a small polyp (less than $5 \mathrm{~mm}$ ) was removed. No ischaemic changes were found in the colonic mucosa.

However, a CT scan showed a renal tumour in the upper part of the left kidney, having signs of bleeding to the urinary tract. Its diameter was $5 \mathrm{~cm}$. Urine cultures and renal function were normal and the patient was symptom-free, as is usually the case in renal tumours.

The preoperative procedures included a renogram (Figure 1) and renal artery embolization (Figure 2).

The patient was scheduled for elective surgery a week later. Laparoscopic nephrectomy was performed under general anaesthesia in the right lateral decubitus position. Pneumoperitoneum was achieved with a Veress needle. Four trocars (2 of $10 \mathrm{~mm}$ and 2 of $5 \mathrm{~mm}$ ) were placed as shown in Figure 3 . The zero degree optical system was applied. Ultracision was used for dissection and closure of the renal artery and the vein was done with vascular clips. The retroperitoneal plane was dissected and after double control of the vessels and ligation of the ureter, the kidney was retrieved via left subcostal incision. No remarkable events were noted during the surgery. Blood loss did not exceed 500 cc during the whole procedure. The only unusual intraoperative findings were firm, fibrotic adhesions between the spleen and

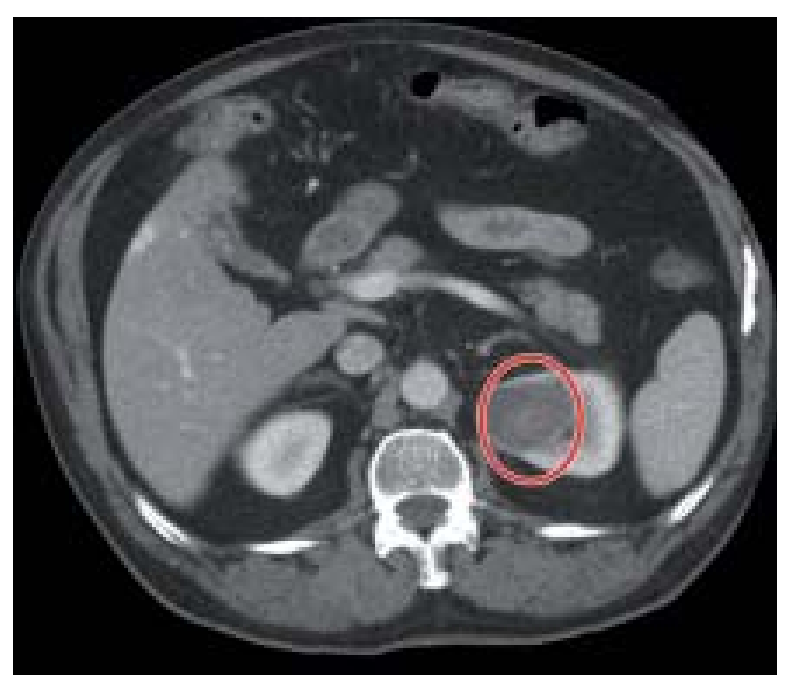

Figure 2. Renal cell cancer 


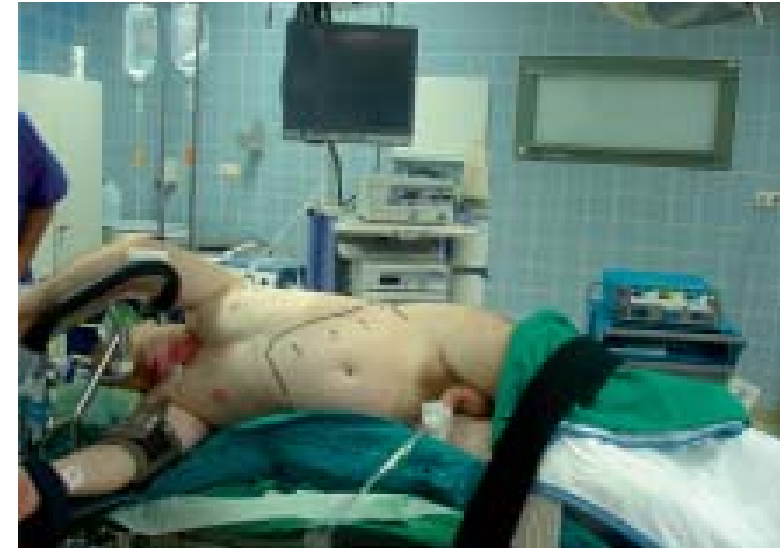

Figure 3. Positioning of the patient on the operating table

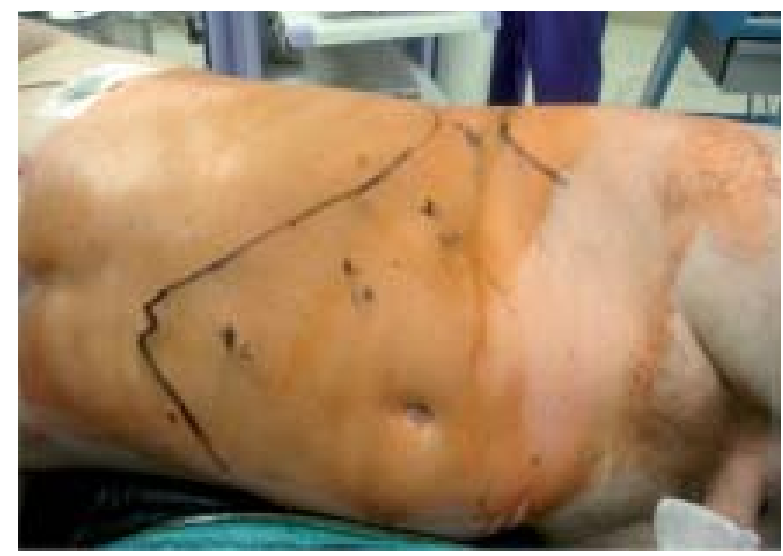

Figure 4. Placement of trocars

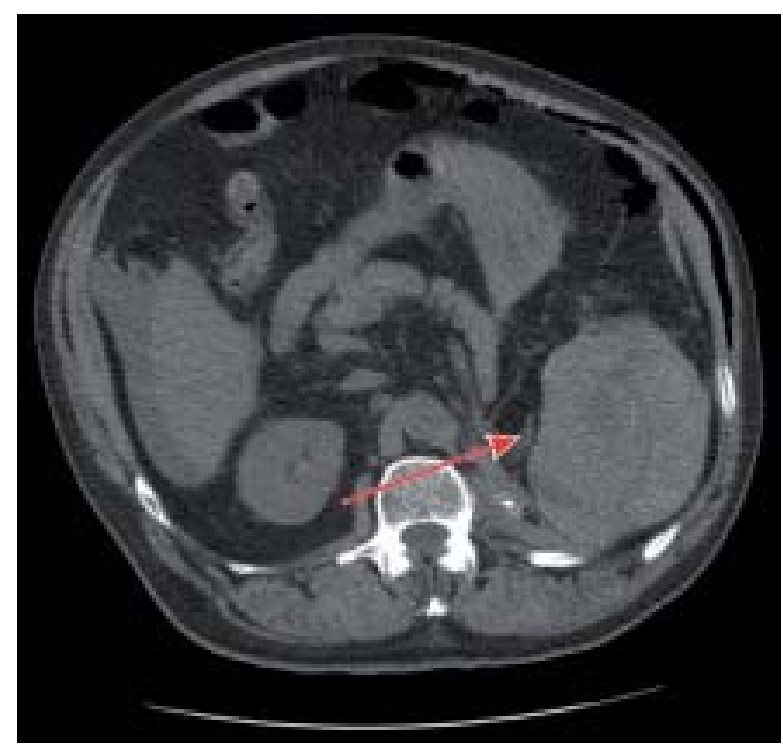

Figure 5. Subcapsular splenic haematoma after surgery the abdominal wall. After removal of the kidney, the spleen was hanging down, suspended on those adhesions and, gradually, a small, assessed as non-relevant subcapsular haematoma started to evolve (Figure 4).

In the recovery room the patient was haemodynamically stable, with less than 50 cc of discharge from the drain. Six h later he developed acute pain and a CT scan was ordered. Radiological images showed that the splenic subcapsular haematoma had increased to $10 \times 10 \mathrm{~cm}$. No free fluid was found at the site of nephrectomy, nor in the peritoneal cavity (Figure 5).

As the patient remained relatively stable and embolization did not seem the best option in a patient with a single kidney, a decision of conservative treatment was made. Unfortunately, $6 \mathrm{~h}$ later emergency surgery had to be performed due to abrupt rupture of the intrasplenic haematoma. The procedure was started with the laparoscopic approach. When massive haemoperitoneum was found and the patient presented with critical hypotension, the surgeon could not achieve the desired pressure of pneumoperitoneum and immediately performed a midline incision, converting to the open procedure. The haemoperitoneum was evacuated and washed out with several litres of saline. Splenic vessels were tied at the hilum. The only visible remnant of the spleen was its capsule, almost completely emptied of the parenchyma. Anatomopathological findings showed normal spleen and splenic capsule.

The patient was discharged 3 weeks later without further complications.

\section{Discussion}

Several hypotheses have been formulated to explain the mechanism of splenic rupture. The spleen is a solid organ that is most often damaged by blunt trauma. The kinetic energy is transmitted through the abdominal wall, causing a lesion, yet intraparenchymal haemorrhage can be contained by the splenic capsule. Spontaneous, non-traumatic rupture of the spleen can occur in both a normal and pathological organ [3].

latrogenic lesion of the spleen is rare, and there are very few cases described of splenic injury during laparoscopic surgery. One of the cases occurred during laparoscopic hysterectomy, when the Veress nee- 
dle produced a subcapsular haematoma of the spleen [4]. Another case was laparoscopic cholecystectomy. The authors speculated that either congenital or posttraumatic adhesions of the parietal peritoneum to the spleen may have caused the capsule to peel off the splenic parenchyma when the pneumoperitoneum was inflated, resulting in subcapsular haematoma and subsequent rupture $[5,6]$.

Although unlikely, iatrogenic injury can be caused by surgical instruments as well. There is a case of splenic injury resulting from the use of a harmonic scalpel during Nissen fundoplication. The authors raised the question whether cavitation produced by the harmonic scalpel could contribute to this type of injury [7].

Splenic trauma is also a recognized complication of both open and laparoscopic renal and adrenal surgery. Most reported injuries occurred intraoperatively with a transperitoneal approach and required immediate management. Left radical nephrectomy is the second most common cause of splenic injury during transabdominal oncological surgery in the upper left quadrant of the abdomen. The incidence of iatrogenic splenectomy during left nephrectomy is estimated to range between $4.3 \%$ and $13.2 \%$. There could be three possible mechanisms of spleen injury: traction, application of retractors or direct trauma from the surgical instruments. Capsular tears, lacerations, avulsions and subcapsular haematomas are the injuries encountered most frequently $[8,9]$.

What makes our case unique is that the spleen remained untouched during the whole procedure. Congenital adhesions of the spleen and emptying of the retroperitoneal space produced the traction on the spleen, which was suspended on the adhesions to the abdominal wall. Due to fragility of the organ, gravity proved to render enough force to cause its injury.

Subcapsular haematoma is neither a predictor of delayed splenic rupture nor an indication for operative management in a haemodynamically stable patient. The degree of parenchymal injury based on CT morphology, and specifically hilar involvement, is crucial in decision making for laparotomy with splenectomy.

We analysed this case thoroughly to avoid similar complications in the future and avoid splenectomy. Preservation of the spleen whenever possible is postulated for its immunological role and splenectomyrelated morbidity, related mainly to subphrenic abscess, pleural effusion and sepsis $[10,11]$. In Spain,

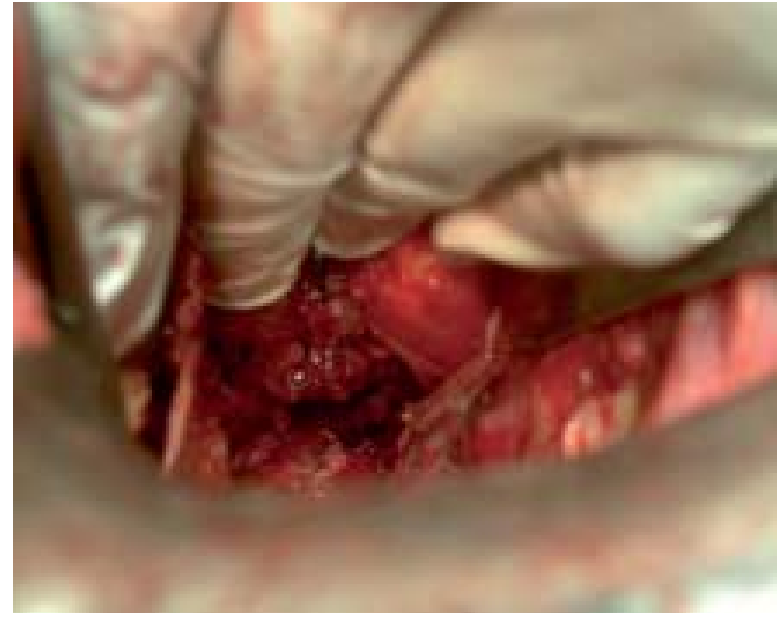

Figure 6. Subcapsular splenectomy being performed. From: Sharma D. Subcapsular splenectomy for delayed spontaneous splenic rupture in a case of sickle cell anaemia. World J Emerg Surg 2009; 4: 17

the conservative treatment rate is around 7-40\% [12]. We think that in a similar case splenorrhaphy with an absorbable mesh to compress the haematoma and drainage to control the postoperative evolution would be our choice of treatment.

Some articles postulate splenorrhaphy as a conservative treatment even in delayed splenic rupture. Mesh of polyglycolic acid with reabsorption time of 85 days, wrapped around the spleen, can facilitate formation of a neocapsule. The main aim of the mesh is to suppress the haemorrhage and avoid splenectomy. All series describe no mortality and feasibility of this technique [13].

When the patient's condition allows, embolization of the splenic artery could prevent surgical intervention. In selected cases, subcapsular splenectomy is also an option (Figure 6).

Assessment of the extent of damage, drainage of the haemoperitoneum, and haemostasis can all be achieved with the laparoscopic approach. Nevertheless, laparoscopic splenectomy in splenic rupture is not easy. Haemostatic fibrin (BioGlue, surgical or Tissucol) can prove useful [14-16]. Haemodynamic stability of the patient, the surgeon's expertise, and availability of equipment all affect safety and feasibility and must be taken into consideration when making a decision $[17,18]$. In each clinical situation the surgeon must weigh the decision individually, with the help of circulatory parameters and CT scan imaging $[19,20]$. 
In conclusion, both acute and delayed splenic rupture demand a knowledgeable and skilled surgeon, who knows how to prevent subcapsular haematoma and splenic rupture in the first place, and how to deal with these complications when they arise.

\section{References}

1. Sarathchandra K. Case report. Mechanisms of delayed splenic rupture: a new hypothesis. Legal Med 2009; 11: S515-7.

2. Ruffolo DC. Delayed splenic rupture: understanding the threat. J Trauma Nurs 2002; 9: 34-40.

3. Kianmanesh R, Aguirre HI, Enjaume F, et al. Ruptures non traumatiques de la rate: trois nouveaux cas et revue de la littérature. Ann Chirurgie 2003; 128: 303-9.

4. Huchon C, Estrade S, Montariol T, et al. Splenic rupture after laparoscopic surgery: a case report. J Minim Invasive Gynecol 2008; 15: 116-8

5. Leff D, Nortley M, Melly L, Bhutiani RP. Ruptured spleen following laparoscopic cholecystectomy. JSLS 2007; 11: 157-60.

6. Takeuchi K, Yamada T, Sato A, et al. Rupture of the spleen as an unusual complication of laparoscopy. A case report. J Reprod Med 2001; 46: 779-80.

7. Morrison JE Jr, Jacobs VR. Rupture of the spleen with the harmonic scalpel: case report of an unexplained complication. JSLS 2007; 11: 268-71.

8. Lai MC, Chang SJ, Chiang IN, Huang CY. Delayed presentation of splenic laceration due to remote trauma in laparoscopic surgery. J Endourol 2008; 22: 705-8.

9. Zieliński H, Pawlicki B, Bortnowski L, et al. Splenic injury during left nephrectomy for renal cell cancer. Pol Merkuriusz Lek 2008; 24: 502-5.

10. Uranus S, Pfeifer J. Non operative treatment of blunt splenic injury. World J Surg 2001; 25: 1405-7.

11. Gomez-Alonso A, Santos Benito FF, Gonzalez-Fernández L, et al. Complicaciones de la esplenectomia. Nuestra casuistica. Cir Esp 2001; 69: 224-30.

12. Martínez Diez M, Ubieto Marínez F, Aguilella Diago V, et al. Criterios cambiantes en la cirugía del bazo. Controversia esplenectomía-cirugía conservadora. Cir Esp 1985; 39: 27-40.

13. Fingerhut $A$, Oberlin $P$, Cote $J$, et al. Spleen salvage using an absorbable mesh: feasibility, realiability and safety. Br J Surg 1992; 79: 325-7.

14. Agarwal N. Laparoscopic splenectomy in a case of blunt abdominal trauma. J Min Access Surg 2009; 5: 78-81.

15. Biggs G, Hafron J, Feliciano J, Hoenig DM. Treatment of splenic injury during laparoscopic nephrectomy with BioGlue, a surgical adhesive. Urology 2005; 66: 882.

16. Orcalli F, Elio A, Veronese E, et al. Conservative laparoscopy in the treatment of posttraumatic splenic laceration using microfiber hemostatic collagen: three case histories. Surg Laparosc Endosc 1998; 8: 445-8.

17. Black JJ, Sinow RM, Wilson SE, Williams RA. Subcapsular hematoma as a predictor of delayed splenic rupture. Am Surg 1992; 58: 732-5.
18. Cresienzo D, Barrat C, Rizk N, Champault G. Videolaparoscopic treatment of splenic injuries. A study of 5 cases. Chirurgie 1998; 123: $600-3$

19. Olmi S, Scaini A, Erba L, et al. Use of fibrin glue (Tissucol) as a hemostatic in laparoscopic conservative treatment of spleen trauma. Surg Endosc 2007; 21: 2051-4.

20. Gayer G, Galperin-Aizenberg M. latrogenic splenic injury in postoperative patients: a series of case reports. Emerg Radiol 2008; 15: 109-13. 